\title{
INFLUÊNCIA DO TIPO DA COBERTURA VEGETAL SOBRE A EROSÃO NO SEMI-ÁRIDO PARAIBANO
}

\author{
Celso Augusto Guimarães Santos ${ }^{1}$, Koichi Suzuki², Masahiro Watanabe ${ }^{3}$ \\ \& Vajapeyam S. Srinivasan ${ }^{4}$
}

\begin{abstract}
RESUMO
O tipo de cobertura vegetal, presente numa área, tem grande influência sobre o escoamento superficial e a produção de sedimentos. O objetivo do presente trabalho é estabelecer uma relação entre o tipo da cobertura vegetal e a erosão do solo nu, mediante uma equação empírica da perda de solo. A equação proposta foi calibrada usando-se dados sintéticos gerados por um modelo físico hidrossedimentológico, cujos parâmetros de erosão foram determinados como valores representativos de uma área desmatada do semi-árido paraibano. Apresenta-se uma comparação entre os valores calculados pela equação e os dados observados em várias parcelas de erosão, as quais apresentavam condições de cobertura vegetal e declividade distintas, localizadas na Bacia Experimental de Sumé, PB. Esta comparação permitiu uma avaliação da influência do tipo de cobertura vegetal sobre a erosão do solo.
\end{abstract}

Palavras-chave: cobertura vegetal, modelo de erosão, semi-árido, produção de sedimentos

\section{INFLUENCE OF THE TYPE OF VEGETAL COVER ON SEDIMENT YIELD IN THE SEMIARID REGION OF PARAÍBA STATE}

\begin{abstract}
The type of vegetation cover present in an area, greatly influences the surface runoff as well as the sediment yield. The objective of this paper is to establish a relationship between the type of vegetal cover and erosion by means of an empirical equation for soil loss. The proposed equation was calibrated using synthetic data obtained from a physically-based runoff-erosion model in which the erosion parameter values are representative of a cleared bare-land surface in the semiarid area of Paraíba State. A comparison between the values obtained from the equation and the observed data collected from several erosion plots in the Sumé Experimental Watershed with different conditions of vegetal cover and slope is presented as an evaluation of the influence of the vegetation cover on soil erosion.
\end{abstract}

Key words: vegetation cover, erosion model, semiarid region, sediment yield

\footnotetext{
Recebido em 16/11/1999, Protocolo 127/99

${ }^{1}$ Professor Adjunto, Dr., Department of Civil and Environmental Engineering, Ehime University, 3-Bunkyo-cho, Matsuyama, Ehime, 790-8577, Japão, Tel./Fax (+81)89-927-9831. E-mail: celso@ dpc.ehime-u.ac.jp

${ }^{2}$ Professor Titular, Dr., Department of Civil and Environmental Engineering, Ehime University. E-mail: ksuzuki@ dpc.ehime-u.ac.jp

${ }^{3}$ Professor Adjunto, Dr., Department of Civil and Environmental Engineering, Ehime University. E-mail: nabemasa@ dpc.ehime-u.ac.jp

${ }^{4}$ Professor Titular, Dr., Departamento de Engenharia Civil, Universidade Federal da Paraíba, Av. Aprígio Veloso 882, Campina Grande, Paraíba, CEP 58109 - 970, Fax: (0xx83) 310 1388. E-mail: srinivas@ rechid.ufpb.br
} 


\section{INTRODUÇÃO}

A cobertura vegetal das bacias hidrográficas sofre várias modificações quer sejam naturais ou artificiais, e estas modificações produzem os mais variados impactos no meio ambiente. $\mathrm{O}$ escoamento superficial é um dos primeiros fatores a serem alterados quando a cobertura vegetal da bacia é modificada e, conseqüentemente, a produção de sedimentos.

Para poder explicar a variação na produção de sedimentos com o tipo de cobertura vegetal, foi desenvolvida uma equação de erosão laminar do tipo da equação proposta por Musgrave (1947). No entanto, é difícil de se dispor da quantidade de dados necessários para o desenvolvimento de uma equação desse tipo, razão por que dados sintéticos produzidos através do modelo físico hidrossedimentológico WESP (Watershed Erosion Simulation Program) desenvolvido por Lopes (1987) foram utilizados. Santos et al. (1994) haviam calibrado o citado modelo para uma microbacia desmatada localizada na Bacia Experimental de Sumé. Dessa forma, a equação pode ser considerada representativa para uma região desmatada do semi-árido paraibano. Utilizando-se esses valores como referência, pode-se fazer comparações com os dados observados em várias parcelas de erosão da mesma bacia experimental, as quais apresentam condições de cobertura vegetal e declividade distintas, para uma avaliação da influência da cobertura vegetal sobre a produção de sedimentos.

Apresentam-se, a seguir, o modelo físico de erosão e escoamento, a geração dos dados sintéticos, a calibração da equação empírica, os dados observados nas parcelas experimentais de erosão, e finalmente a avaliação comparativa entre os valores previstos pela equação desenvolvida para áreas desmatadas e os dados observados em diferentes parcelas de erosão, com diversos tipos de cobertura vegetal.

\section{MATERIAL E MÉTODOS}

\section{O modelo hidrodinâmico}

O modelo escolhido a ser aplicado neste trabalho foi o modelo WESP, pois o mesmo foi desenvolvido para simular a resposta hidrossedimentológica de pequenas bacias e já foi calibrado com sucesso para uma região semi-árida nordestina. O modelo, sendo do tipo hidrodinâmico, oferece a possibilidade de sucesso quando aplicado a uma ampla faixa de condições e ajuda na compreensão dos processos naturais possibilitando, assim, uma ponte entre a teoria e a aplicação hidrológica (Lopes \& Lane, 1987). O modelo usa a equação de Green-Ampt modificada por Mein \& Larson (1973) para modelar a infiltração. Esta equação fornece, para a taxa de infiltração, a expressão (Lopes, 1987):

$$
\mathrm{f}(\mathrm{t})=\mathrm{K}_{\mathrm{S}}\left(1+\frac{\mathrm{N}_{\mathrm{S}}}{\mathrm{F}(\mathrm{t})}\right)
$$

onde $\mathrm{f}(\mathrm{t})$ é a taxa de infiltração $\left(\mathrm{m} \mathrm{s}^{-1}\right), \mathrm{K}_{\mathrm{S}}$ é a condutividade hidráulica do solo $\left(\mathrm{m} \mathrm{s}^{-1}\right), \mathrm{N}_{\mathrm{s}}$ é o parâmetro do potencial de capilaridade na frente de molhamento $(\mathrm{m}), \mathrm{F}(\mathrm{t})$ é a altura acumulada de água infiltrada (m) e t é a variável tempo (s).

O fluxo superficial é considerado variado e unidimensional e a equação para a velocidade do escoamento superficial nos planos pode ser obtida a partir da aproximação da onda cinemática, em que a declividade da linha de energia é considerada igual à declividade do plano $\left(\mathrm{S}_{\mathrm{f}}=\mathrm{S}_{0}\right)$. Esta aproximação é válida quando os efeitos da gravidade e de atrito dominam sobre os outros fatores na equação da quantidade de movimento, resultando numa relação simples entre a velocidade e a profundidade do fluxo, na forma:

$$
\mathrm{u}=\alpha^{\prime} \mathrm{h}^{\mathrm{m}-1}
$$

onde h é a espessura da lâmina de fluxo (m), $\alpha^{\prime}$ é um parâmetro de rugosidade da superfície e m é um parâmetro geométrico. A combinação da Eq. (2) com a equação de Manning para escoamento turbulento sobre planos de grande largura resulta em:

$$
\begin{gathered}
\alpha^{\prime}=(1 / n) \mathrm{S}_{0}{ }^{1 / 2} \\
\mathrm{~m}=5 / 3
\end{gathered}
$$

A produção de sedimentos é igual à erosão menos a deposição. A erosão por sua vez é devido ao impacto das gotas da chuva e devido à força de cisalhamento do escoamento. Dessa forma, o fluxo de sedimento $\Phi$ pode ser expresso por:

$$
\Phi=\mathrm{e}_{1}+\mathrm{e}_{\mathrm{R}}-\mathrm{d}
$$

onde $e_{\mathrm{I}}$ é a taxa de desprendimento de sedimentos pelo impacto da chuva, $e_{R}$ é a taxa de desprendimento de sedimentos pela força de cisalhamento, e dé a taxa de deposição de sedimentos. A taxa de desprendimento $\mathrm{e}_{\mathrm{I}}\left(\mathrm{kg} \mathrm{m}^{-2} \mathrm{~s}^{-1}\right)$ é dada por:

$$
\mathrm{e}_{1}=\mathrm{K}_{1} \mathrm{Ir}_{\mathrm{e}}
$$

onde $\mathrm{K}_{\mathrm{I}}$ é um parâmetro de destacamento do solo pelo impacto da chuva $\left(\mathrm{kg} \mathrm{s} \mathrm{m}^{-4}\right)$, I é a intensidade da chuva $\left(\mathrm{m} \mathrm{s}^{-1}\right)$, e $\mathrm{r}_{\mathrm{e}}$ é a intensidade de chuva efetiva $\left(\mathrm{m} \mathrm{s}^{-1}\right)$. A taxa de erosão $e_{R}$ $\left(\mathrm{kg} \mathrm{m}^{-2} \mathrm{~s}^{-1}\right)$ é expressa por:

$$
\mathrm{e}_{\mathrm{R}}=\mathrm{K}_{\mathrm{R}} \tau^{1.5}
$$

onde $\mathrm{K}_{\mathrm{R}}$ é um fator de destacamento pela tensão de cisalhamento do escoamento ( $\mathrm{kg} \mathrm{m} \mathrm{N}^{-1.5} \mathrm{~s}^{-1}$ ) e $\tau$ é a tensão de cisalhamento efetiva $\left(\mathrm{N} \mathrm{m}^{-2}\right)$ dada por:

$$
\tau=\gamma R_{H} S_{f}
$$

onde $\gamma$ é o peso específico da água $\left(\mathrm{N} \mathrm{m}^{-3}\right)$ e $\mathrm{R}_{\mathrm{H}}$ é o raio hidráulico do fluxo igual à profundidade do fluxo, para grandes larguras. Finalmente, a taxa de deposição de sedimentos $\mathrm{d}\left(\mathrm{kg} \mathrm{m}^{-2} \mathrm{~s}^{-1}\right)$ é dada por:

$$
\mathrm{d}=\varepsilon \mathrm{V}_{\mathrm{s}} \mathrm{c}
$$

onde $\varepsilon$ é um coeficiente que depende das propriedades do solo e fluido admitido ser igual a 0,5 para planos (Lopes, 1987), c(x,t) é a concentração de sedimentos em transporte $\left(\mathrm{kg} \mathrm{m}^{-3}\right), \mathrm{e} \mathrm{V}_{\mathrm{s}}$ é a velocidade de queda da partícula $\left(\mathrm{m} \mathrm{s}^{-1}\right)$ calculada pela equação de Rubey (Raudikivi, 1976): 


$$
\mathrm{V}_{\mathrm{s}}=\mathrm{F}_{\mathrm{o}} \sqrt{\frac{\left(\gamma_{\mathrm{s}}-\gamma\right)}{\gamma} \mathrm{gd}_{\mathrm{s}}}
$$

na qual

$$
\mathrm{F}_{\mathrm{o}}=\sqrt{\frac{2}{3}+\frac{36 v^{2}}{\operatorname{gd}_{\mathrm{s}}^{3}\left(\frac{\gamma_{\mathrm{s}}}{\gamma}-1\right)}}-\sqrt{\frac{36 v^{2}}{\operatorname{gd}_{\mathrm{s}}^{3}\left(\frac{\gamma_{\mathrm{s}}}{\gamma}-1\right)}}
$$

onde $\gamma_{\mathrm{s}}$ é o peso específico do sedimento $\left(\mathrm{N} \mathrm{m}^{-3}\right), \mathrm{v}$ é a viscosidade cinemática da água $\left(\mathrm{m}^{2} \mathrm{~s}^{-1}\right), \mathrm{d}_{\mathrm{s}}$ é o diâmetro médio do sedimento (m) e $g$ é a aceleração da gravidade $\left(\mathrm{m} \mathrm{s}^{-2}\right)$.

\section{Dados sintéticos}

Os dados sintéticos de erosão foram gerados admitindo-se chuvas de intensidades diferentes sobre parcelas hipotéticas de um metro de largura, de comprimentos de 30, 100 e $300 \mathrm{~m}$ e com declividades de $4 \%, 10 \%$ e $20 \%$, perfazendo um conjunto de nove parcelas. As intensidades das chuvas aplicadas nessas parcelas foram de 10,20 e $50 \mathrm{~mm} \mathrm{~h}^{-1}$ e os valores da produção de sedimentos em cada parcela, quando submetida às diversas intensidades das chuvas, foram calculados pelo modelo WESP, exposto no subitem anterior. Os parâmetros de erosão foram aqueles calibrados para a região semi-árida paraibana, que constituem o parâmetro de destacamento do solo pelo impacto da chuva $\mathrm{K}_{\mathrm{I}}$, presente na Eq. (5), e o parâmetro de destacamento pela tensão de cisalhamento do escoamento $\mathrm{K}_{\mathrm{R}}$, na Eq. (6).

Santos et al. (1994) determinaram os valores médios para esses parâmetros $\left(\mathrm{K}_{\mathrm{I}}\right.$ e $\left.\mathrm{K}_{\mathrm{R}}\right)$, como sendo $5,1 \times 10^{8} \mathrm{~kg} \mathrm{~s} \mathrm{~m}^{-4}$ e $2,1 \mathrm{~kg} \mathrm{~m} \mathrm{~N}^{-1,5} \mathrm{~s}^{-1}$, respectivamente. Estes valores foram obtidos mediante calibração do modelo para uma microbacia desmatada da Bacia Experimental de Sumé de área igual a 0,48 ha, perímetro de $302 \mathrm{~m}$, declividade igual a 7,1\% e diâmetro médio dos sedimentos igual a $0,50 \mathrm{~mm}$.

Para os parâmetros de infiltração foi admitido o valor de $5 \mathrm{~mm} \mathrm{~h}^{-1}$ para a condutividade hidráulica saturada do solo $\mathrm{K}_{\mathrm{S}}$ (Eq. 1) baseado em testes de campo, e o valor zero para o parâmetro do potencial de capilaridade na frente de molhamento
$\mathrm{N}_{\mathrm{S}}$, na Eq. (1) para o estado de saturação. Outro valor que deve ser mencionado aqui é o coeficiente de rugosidade da equação de Manning, n, assumido igual a 0,02, baseado no tipo do solo, tamanho dos sedimentos e característica da superfície.

Uma vez tendo-se as dimensões das parcelas, intensidades das chuvas e valores dos parâmetros de erosão, o programa WESP foi utilizado para obter os valores da produção de sedimentos E. A Figura 1 (A, B, C) apresenta os valores simulados pelo modelo WESP para as diversas condições de comprimento da parcela, intensidade de chuva e declividade da parcela.

\section{Calibração da equação empírica}

Musgrave (1947) sintetizou os resultados de análises de medições da perda de solo sob diversas condições e expressou a taxa de perda de solo E por uma equação empírica:

$$
E=\alpha L^{\beta 1} I^{\beta 2} S_{0}^{\beta 3}
$$

onde $\alpha$ é um parâmetro de cobertura e erodibilidade inerente do solo, $\beta_{1}, \beta_{2}$ e $\beta_{3}$ são expoentes constantes, Lé o comprimento da rampa, $\mathrm{S}_{0}$ é a declividade, e I é a intensidade da chuva, conforme descritos anteriormente.

Para se determinar os expoentes e o parâmetro $\alpha$ desta equação, se faria necessário a coleta de um número muito grande de dados observados para a área desejada, o que às vezes se torna uma tarefa impossível ou fora da realidade de várias regiões, principalmente nos países em desenvolvimento, devido ao alto custo de coleta de dados de campo. Com o intuito de simplificar tal tarefa, dados sintéticos foram gerados baseados nos processos e propriedades físicas, apresentados no subitem "O modelo hidrodinâmico".

Os expoentes da equação foram então determinados através do ajustamento da Eq. (11) aos dados sintéticos obtidos através da simulação usando o modelo WESP, conforme apresentado na Figura 1A, B e C. Os expoentes $\beta_{1}, \beta_{2}$ e $\beta_{3}$ são as declividades das linhas retas obtidas na Figura 1 e a equação final obtida foi:

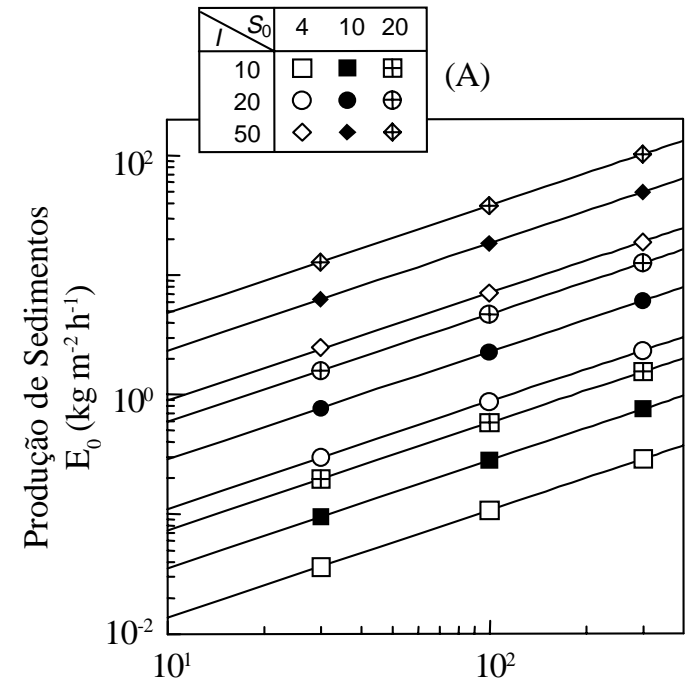

Comprimento L (m)

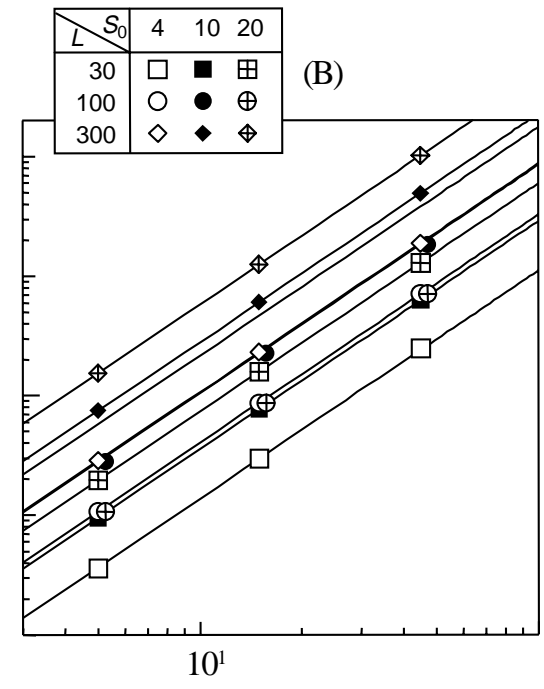

Intensidade de Chuva $\mathrm{r}_{\mathrm{e}}\left(\mathrm{mm} \mathrm{h}^{-1}\right)$

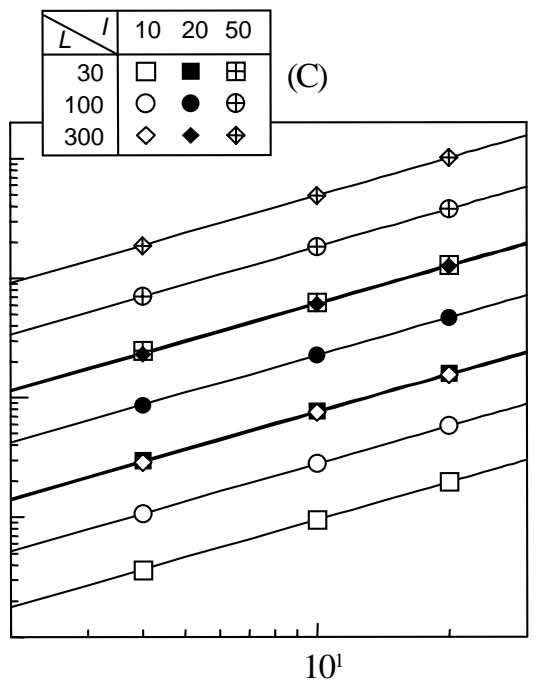

Declividade $\mathrm{S}_{0}(\%)$

Figura 1. Relação entre a produção de sedimentos simulada e comprimento de rampa L (A), intensidade da chuva efetiva $r_{e}(B)$, declividade $\mathrm{S}_{0}(\mathrm{C})$ 


$$
\mathrm{E}=1,91 \times 10^{-5} \mathrm{~L}^{0,9} \mathrm{r}_{\mathrm{e}}^{1,91} \mathrm{~S}_{0}^{1,04}
$$

onde E está em $\mathrm{kg} \mathrm{m}^{-2} \mathrm{~h}^{-1}$, L em m, $\mathrm{S}_{0}$ em percentagem, e $\mathrm{r}_{\mathrm{e}}$ é a intensidade da chuva efetiva em $\mathrm{mm} \mathrm{h}^{-1}$, dada por $\mathrm{r}_{\mathrm{e}}=\mathrm{I}-\mathrm{K}_{\mathrm{S}}$, sendo $\mathrm{K}_{\mathrm{s}}=5 \mathrm{~mm} \mathrm{~h}^{-1}$, o que é a taxa final de infiltração ou condutividade hidráulica efetiva do solo saturado. De forma alternativa, a Eq. (12) pode ser expressa em termos da intensidade da chuva total $I\left(\mathrm{~mm} \mathrm{~h}^{-1}\right)$ como:

$$
\mathrm{E}=1,19 \times 10^{-6} \mathrm{~L}^{0,9} \mathrm{I}^{2,59} \mathrm{~S}_{0}^{1,04}
$$

\section{Parcelas experimentais}

As parcelas experimentais foram instaladas na Bacia Experimental de Sumé localizada no Estado da Paraíba, operada pela SUDENE, ORSTOM e UFPB (Cadier et al., 1983). As parcelas têm $100 \mathrm{~m}^{2}$, sendo 4,55 m de largura e 22,5 m de comprimento. As declividades, os anos de instalação e tipos de cobertura das parcelas estão apresentados na Tabela 1. O mesmo tipo de manejo e vegetação foram mantidos entre as parcelas $1 \mathrm{e} 4$, parcelas $2 \mathrm{e} 3$, e posteriormente parcelas 5 e 9 a fim de permitir uma comparação dos resultados para diferentes declividades. Encontram-se também, nesta bacia experimental, 4 microbacias com áreas em torno de $0,5 \mathrm{ha}$, sendo duas totalmente desmatadas e outras duas com caatinga nativa; o tipo do solo encontrado na bacia experimental é "Bruno não Cálcico-Vértico".

Tabela 1. Características das parcelas de erosão

\begin{tabular}{cclc}
\hline Parcela & $\begin{array}{c}\text { Declividade } \\
\text { Média (\%) }\end{array}$ & Cobertura Vegetal & $\begin{array}{c}\text { Ano de } \\
\text { Instalação }\end{array}$ \\
\hline 1 & 3,8 & Desmatada & 1982 \\
2 & 3,9 & Cobertura morta & 1982 \\
3 & 7,2 & Cobertura morta & 1982 \\
4 & 7,0 & Desmatada & 1982 \\
5 & 9,5 & Caatinga nativa & 1982 \\
6 & 4,0 & Palma morro abaixo & 1983 \\
7 & 4,0 & Palma em contorno & 1983 \\
8 & 4,0 & Desmatada e revolvida & 1986 \\
9 & 4,0 & Caatinga nova (1981) & 1986 \\
\hline
\end{tabular}

Nas parcelas desmatadas (parcelas 1 e 4), a vegetação era removida quando estava atingindo $5 \mathrm{~cm}$, ao passo que nas parcelas com cobertura morta (parcelas 2 e 3 ) a vegetação era cortada quando estava atingindo 20 a $25 \mathrm{~cm}$, mas deixada na própria parcela; a parcela 8 foi mantida sem vegetação e seu solo revolvido freqüentemente. As parcelas 6 e 7, com palmas, foram limpas quando a vegetação estava chegando a $5 \mathrm{~cm}$ de altura. A parcela 5 com vegetação nativa não sofreu intervenção alguma, e a parcela 9 também não sofreu mais intervenção a partir de 1981.

Baseando-se na experiência de um trabalho anterior (Santos et al., 1994) vários eventos foram escolhidos entre 1987 e 1988. As relações observadas entre o escoamento e a chuva encontram-se na Figura 2A e B para as parcelas com e sem vegetação, respectivamente, pode-se observar que apesar das tendências semelhantes entre os dois casos, as parcelas providas de vegetação apresentam um maior número de eventos com pequeno escoamento, isto é, menos que 0,1 $\mathrm{mm}$. A Figura 3A e B mostram a relação entre a produção de sedimentos observados $\mathrm{E}_{\mathrm{o}} \mathrm{e}$ a intensidade da chuva I, também para as parcelas com e sem vegetação, de onde pode-se observar que quando a proteção do solo é mínima, a erosão

é máxima. Tanto a vegetação nativa (caatinga) quanto a cobertura morta, mostram-se eficientes para controlar a erosão superficial.

(A)

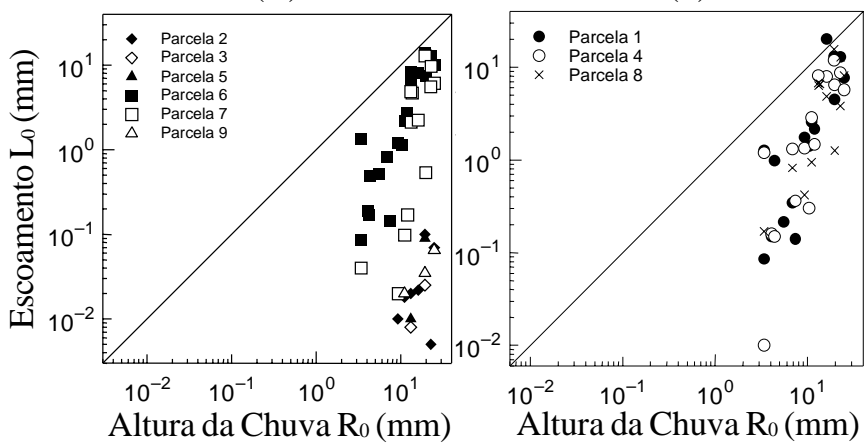

Figura 2. Relação entre o escoamento observado $L_{o}$ e a altura de chuva observada $R_{o}$ para as parcelas com cobertura vegetal (A), solo nu (B)
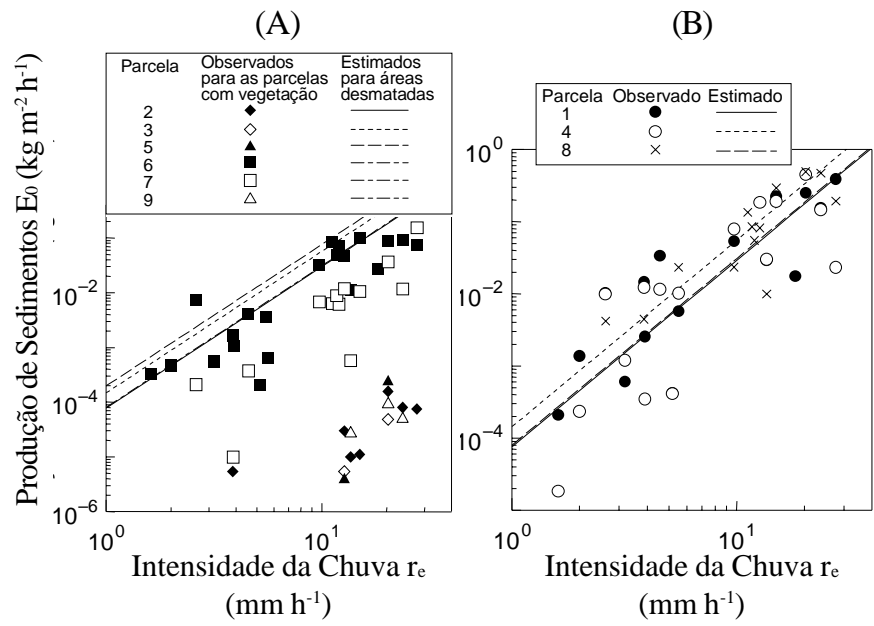

Figura 3. Relação entre a produção de sedimento observada $\mathrm{E}$ e a intensidade efetiva da chuva $r_{e}$ para as parcelas com cobertura vegetal (A), solo nu (B)

\section{RESULTADOS E DISCUSSÃO}

Por ser uma equação empírica desenvolvida usando-se dados obtidos através de um modelo cujos parâmetros foram calibrados para uma região semi-árida nordestina desmatada, ela pode ser considerada representativa para tal área. Dessa forma, o uso da mesma para comparação com dados observados em nove parcelas experimentais de Sumé permitiria uma avaliação da influência da cobertura vegetal sobre a erosão.

As simulações feitas usando a Eq. (12) estão representadas pelas linhas retas na Figura 3A e B. Nas figuras, as retas se distinguem pela declividade da parcela, pois o comprimento do plano é o mesmo para todas as parcelas. Pela Figura 3B fica evidente que existe uma boa conformidade entre os valores observados e os calculados para as parcelas sem vegetação, apesar de uma notável dispersão. A dispersão dos pontos em torno das retas pode ser explicada pelo fato de que a Eq. (12) considera uma chuva efetiva de intensidade uniforme igual à intensidade média do evento e, na prática, a variação da intensidade durante a chuva provoca uma variação da produção dos sedimentos ao longo do evento. Pode-se observar que a 
declividade maior da parcela 4 (7\%) promove maior erosão em relação à parcela $1(3,8 \%)$ enquanto a parcela $8(4 \%)$ produz tanto quanto a parcela 4 , devido ao manejo da parcela 8 , em que o solo foi periodicamente revolvido (arado). Assim, o aumento da erosão pela alta disponibilidade de sedimentos desprendidos é compensado pela diminuição do transporte, devido à menor declividade da parcela 8. Entretanto, observa-se que na Figura $3 \mathrm{~A}$, para as parcelas com cobertura vegetal, os dados de algumas parcelas também se aproximam da condição da parcela 1 (desmatada) como é o caso da parcela 6 com palma. Isto significa que alguns tipos de cobertura vegetal são ineficientes em proteger o solo contra a erosão. Assim, pode ser observado que as palmas plantadas morro abaixo, o qual é um tipo de cultivo muito praticado no nordeste brasileiro, não protege o solo; por outro lado, o melhor tipo de cobertura vegetal, entre os estudados, mostra-se ser a vegetação nativa, a caatinga, outro ponto importante de ser observado é que um decréscimo significativo na produção de sedimentos pode ser, de uma forma geral, obtido quando o solo está protegido pela cobertura morta.

O parâmetro $\alpha$ serve para uma avaliação comparativa. Este, além de ser um parâmetro de erodibilidade inerente do solo, serve também, para caracterizar o tipo de cobertura do solo. A Figura 4 mostra a relação entre o parâmetro $\alpha$ e o tipo de vegetação, e dela pode-se observar que para as parcelas desmatadas tal parâmetro varia entre $10^{-6} \mathrm{e} 10^{-8}$ enquanto para as parcelas com vegetação nativa o mesmo varia entre $10^{-8} \mathrm{e}$ $10^{-10}$. As parcelas com palmas mostram uma variação similar às das parcelas desmatadas, mas a parcela com palmas em contorno apresenta uma pequena diminuição no valor do mesmo. As parcelas 4 e 8 são ambas desmatadas, porém de diferentes declividades. A parcela 8 teve seu solo revolvido, enquanto a parcela 4 era apenas desmatada, mas com maior declividade do que da parcela 8 . As duas parcelas ficaram caracterizadas pelo mesmo valor de $\alpha$.

As parcelas com cobertura morta e as com caatinga mostram, apesar das diferenças de declividades, variações da erosão muito pequenas e apresentam valores do parâmetro $\alpha$ bastante próximos. Desta forma, o valor médio ou a faixa da

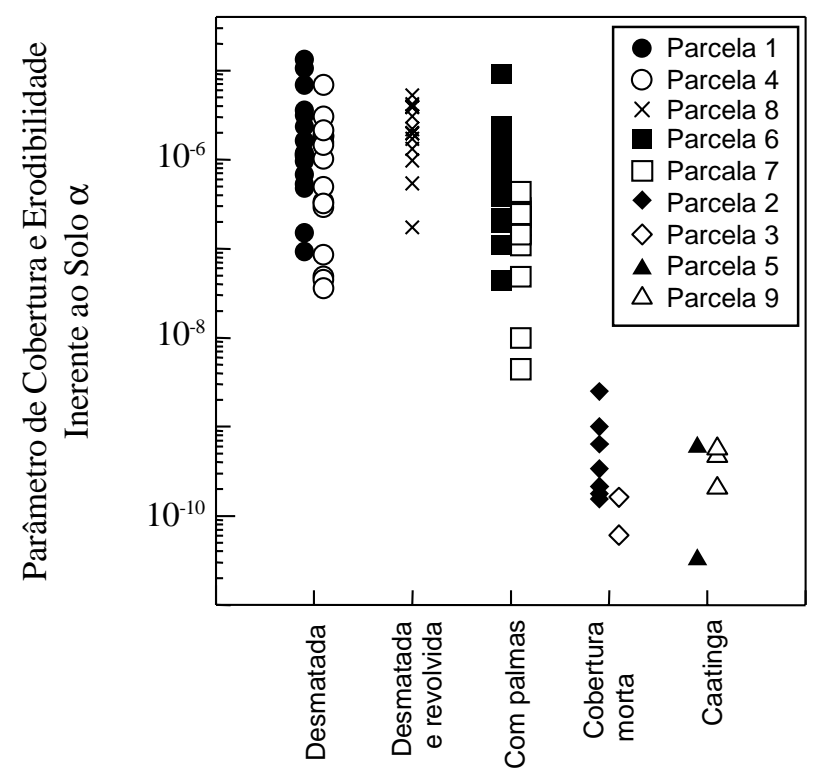

Figura 4. Relação entre o parâmetro de cobertura e erodibilidade inerente ao solo $\alpha$ e tipo de cobertura vegetal variação de $\alpha$ serve como bom indicador do efeito da influência da cobertura vegetal sobre a erosão ou a produção de sedimentos.

\section{CONCLUSÕES}

1. A equação para predizer a taxa de erosão laminar $\mathrm{E}\left(\mathrm{kg} \mathrm{m}^{-2} \mathrm{~h}^{-1}\right)$ para uma região semi-árida paraibana pode ser expressa pela seguinte equação:

$$
\mathrm{E}=1,91 \times 10^{-5} \mathrm{~L}^{0,9} \mathrm{r}^{1,91} \mathrm{~S}_{0}^{1,04}
$$

onde L é o comprimento da rampa (m), $\mathrm{S}_{0}$ é a declividade (\%), e $\mathrm{r}_{\mathrm{e}}$ é a intensidade da chuva efetiva $\left(\mathrm{mm} \mathrm{h}^{-1}\right)$.

2. Quando o solo semi-árido paraibano está protegido pela vegetação nativa ou pela cobertura morta, a produção de sedimentos diminui significativamente. A efetividade da palma para proteger o solo contra a erosão, porém, é insignificante.

3. A prática de cultivo morro abaixo é danosa e aumenta a erosão do solo.

4. O valor do parâmetro de cobertura e erodibilidade $\alpha$ para as parcelas desmatadas ficou entre $10^{-6}$ e $10^{-8}$, mas para as parcelas com vegetação seu valor variou entre $10^{-8} \mathrm{e} 10^{-10}$, indicando uma grande influência do desmatamento sobre o processo de erosão do solo.

\section{AGRADECIMENTOS}

O trabalho de campo incluído neste trabalho foi realizado pela SUDENE, UFPB e ORSTOM (França). Agradecimentos também vão ao Professor Dr. Vicente L. Lopes da Universidade do Arizona (EUA) por fornecer o programa WESP.

\section{REFERÊNCIAS BIBLIOGRÁFICAS}

CADIER, E.; FREITAS, B.J.; LEPRUN, J.C. Bacia experimental de Sumé, instalação e primeiros resultados. Recife: SUDENE, Série Hidrologia, v. 16, 1983. 88p.

LOPES, V.L. A numerical model of watershed erosion and sediment yield. Tucson: University of Arizona, 1987. 148p. $\mathrm{Ph} . \mathrm{D}$. Thesis

LOPES, V.L.; LANE, L.J. Um modelo computacional de simulação hidrológica. In: SIMPÓSIO BRASILEIRO DE RECURSOS HÍDRICOS, 7, v. 2, 1987, Salvador. Anais... Salvador: Associação Brasileira de Recursos Hídricos, 1987. p. 481494.

MEIN, R.G.; LARSON, C.L. Modeling infiltration during a steady rain. Water Resources Research, Washington, v. 9, n. 2, p. 384-394, 1973.

MUSGRAVE, G.W. The quantitative evaluation of factors in water erosion: A first approximation. Journal Soil Water Conservation, Iowa, v. 2, n. 3, p. 133-138, 1947.

RAUDIKIVI, A.J. Loose boundary hydraulics. 2.ed. Oxford: Pergamon Press, 1976.397p.

SANTOS, C.A.G.; SUZUKI, K.; WATANABE, M.; SRINIVASAN, V.S. Optimization of coefficients in runofferosion modeling by Standardized Powell method. Journal of Hydroscience and Hydraulic Engineering, JSCE, Tokyo, v. 12, n. 1, p. 67-78, 1994. 